\title{
Cost-Utility Analysis of the Oral Fluoropyrimidine S-1 Versus Conventional Intravenous Chemotherapy in Advanced or Recurrent Gastric Cancer
}

\author{
Hiroyuki Sakamaki ${ }^{*}$, , Shunya Ikeda ${ }^{2}$, Shuichi Yajima ${ }^{3}$, Naoki Ikegami ${ }^{3}$, Katsumi Tanaka ${ }^{4}$, \\ Hisanori Shimizu ${ }^{4}$ and Jun-ichiro Murayama ${ }^{4}$ \\ ${ }^{I}$ Meijo University, Faculty of Pharmacy, Nagoya-shi, Aichi, Japan \\ ${ }^{2}$ International University of Health and Welfare School of Pharmacy, Ohtawara-shi, Tochigi, Japan \\ ${ }^{3}$ Keio University School of Medicine, Shinjuku-ku, Tokyo, Japan \\ ${ }^{4}$ Showa University Hospital, Shinagawa-ku, Tokyo, Japan
}

\begin{abstract}
Objective: The aim of this study was to compare the cost-utility of S-1, an oral anticancer agent developed in Japan, and conventional intravenous chemotherapy in patients with advanced or recurrent gastric cancer on the basis of the cost and quality of life (QOL) data we previously reported.

Methods: Patients with advanced or recurrent gastric cancer who could ingest food were identified retrospectively from the ordering system database of Showa University Hospital between January 1998 and July 2001. Costs incurred during chemotherapy were calculated on the basis of hospital billing data. The utilities of chemotherapy were assessed by oncology pharmacists and nurses on the basis of the patients' medical records. Cost-utility analysis was conducted from a societal perspective.
\end{abstract}

Results: Of the 23 patients who met the inclusion criteria, 13 received S-1 (S-1 group) and 10 received conventional intravenous chemotherapy (IV chemotherapy group). The average ( \pm SE) monthly cost during chemotherapy was significantly lower in the S-1 group $(327,640 \pm 47,647$ yen) than in the IV chemotherapy group $(852,874 \pm 62,412$ yen). Average $( \pm$ SE) utilities in the S-1 group $(0.84 \pm 0.02-0.94 \pm 0.01)$ were significantly higher than those in IV chemotherapy group $(0.52 \pm 0.04-0.79 \pm 0.02)$.

Conclusion: S-1 is a dominant strategy with lower costs and better health outcomes than conventional intravenous chemotherapy in patients with advanced or recurrent gastric cancer.

\section{INTRODUCTION}

Lung, colorectal and breast cancers are the common form of malignancy in northern America and Europe [1]. However, gastric cancer has long been the leading incidence of cancer in Japan [2]. The treatment strategy for gastric cancer is surgery for early disease and chemotherapy for advanced or recurrent disease, similar to other types of cancer. Combination regimens including fluorouracil are widely used as standard regimen to treat advanced and recurrent gastric cancer [3]. In Japan, combination chemotherapy including continuous intravenous infusion of fluorouracil, such as fluorouracil plus cisplatin or methotrexate, has long been used [4].

S-1 (TS-1, Taiho Pharmaceutical) is an oral anticancer agent that combines tegafur, a prodrug of fluorouracil, with gimeracil, an inhibitor of the degradation of fluorouracil, and oteracil, an inhibitor of the phosphorylation of fluorouracil in the gastrointestinal tract. S-1 was launched in Japan in 1999. The response rate with S-1 alone exceeded $40 \%$ in two late

*Address correspondence to this author at the Department of Pharmacy, Meijo University, 150, Yagotoyama, Tempaku-ku, Nagoya-shi, 468-8503, Japan; Tel: +81 52839 2668; E-mail: sakamaki@ccmfs.meijo-u.ac.jp phase II trials in patients with advanced or recurrent gastric cancer $[5,6]$. A randomized clinical trial (JCOG 9912) showed that oral S-1 and standard treatment with intravenous 5-fluorouracil have similar survival for gastric cancer [7]. S1 is now widely used for the management of advanced or recurrence gastric cancer in Japan and is also being paid attention to overseas [8].

Apart from greater convenience, oral preparations are generally considered more cost effective and better tolerated than intravenous treatment. To confirm this assumption, we conducted a cost-utility analysis to compare treatmentrelated costs and the quality of life (QOL) between S-1 and conventional intravenous chemotherapy in patients with advanced or recurrent gastric cancer on the basis of the our previous studies [9-11].

\section{MATERIALS AND METHODOLOGY}

\section{Type of Analysis}

The cost-utility analysis for S-1 in comparison with conventional intravenous chemotherapy was conducted from a societal perspective.

We expressed the costs and utilities on a per-month basis, since we considered that the survival of S-1 treatment and 
Table 1. Treatment Schedules and Outcome Results

\begin{tabular}{|c|c|c|c|}
\hline & Treatment Schedule & Response Rate (\%) & MST(M) \\
\hline S-1 $[5,6]$ & tegafur $80-120 \mathrm{mg} /$ body on days $1-28$ of a 42 -day cycle & $44-49$ & 7.4-8.9 \\
\hline low dose FP [18] & 5 -FU $350 \mathrm{mg} / \mathrm{m}^{2}$ on days $1-28$ plus cisplatin $10 \mathrm{mg} / \mathrm{m}^{2}$ on days $1-5$ of a 28 -day cycle & 50 & 5.5 \\
\hline MTX/5FU [19] & methotrexate $100 \mathrm{mg} / \mathrm{m}^{2}$ plus 5 -FU $600 \mathrm{mg} / \mathrm{m}^{2}$ weekly & 40.5 & 7.6 \\
\hline $\mathrm{CPT} / \mathrm{P}[20]$ & irinotecan $70 \mathrm{mg} / \mathrm{m}^{2}$ on days 1,15 plus cisplatin $80 \mathrm{mg} / \mathrm{m}^{2}$ on day 1 of a 28 -day cycle & 48 & 9.7 \\
\hline
\end{tabular}

that of conventional intravenous chemotherapy are essentially the same. The response rates of S-1 and several intravenous regimens we assessed in this study are shown in Table 1.

\section{Patient Selection}

The patients with advanced or recurrent gastric cancer were selected for this analysis from the patient database of Showa University Hospital during the period from 1998 to 2001 (Fig. 1).

To avoid bias in selecting the eligible patients for the analysis, patients who met the following criteria were studied. For S-1, all patients who started treatment after August 1999, when S-1 became available at the hospital, were studied. For conventional intravenous chemotherapy, all patients who received injectable preparations of 5-fluorouracil, cisplatin, methotrexate, or irinotecan before August 1999 and were able to receive oral drugs as confirmed by their physicians were studied.

\section{Investigation of Medical Resource Utilization and Costs}

The medical resource utilization data were collected from the Showa University Hospital order entry system during the period from January 1998 to July 2001.

The following costs were calculated on the basis of the Japanese National Health Insurance fee-for-service system: costs related to hospitalization and physician consultation (health care professional visits); costs for laboratory and diagnostic tests; costs for drugs (injections, oral drugs, administration); costs for operations and procedures (including transfusion), and other costs. Because the treatments for all of the patients ended within one year, we didn't discount the costs.

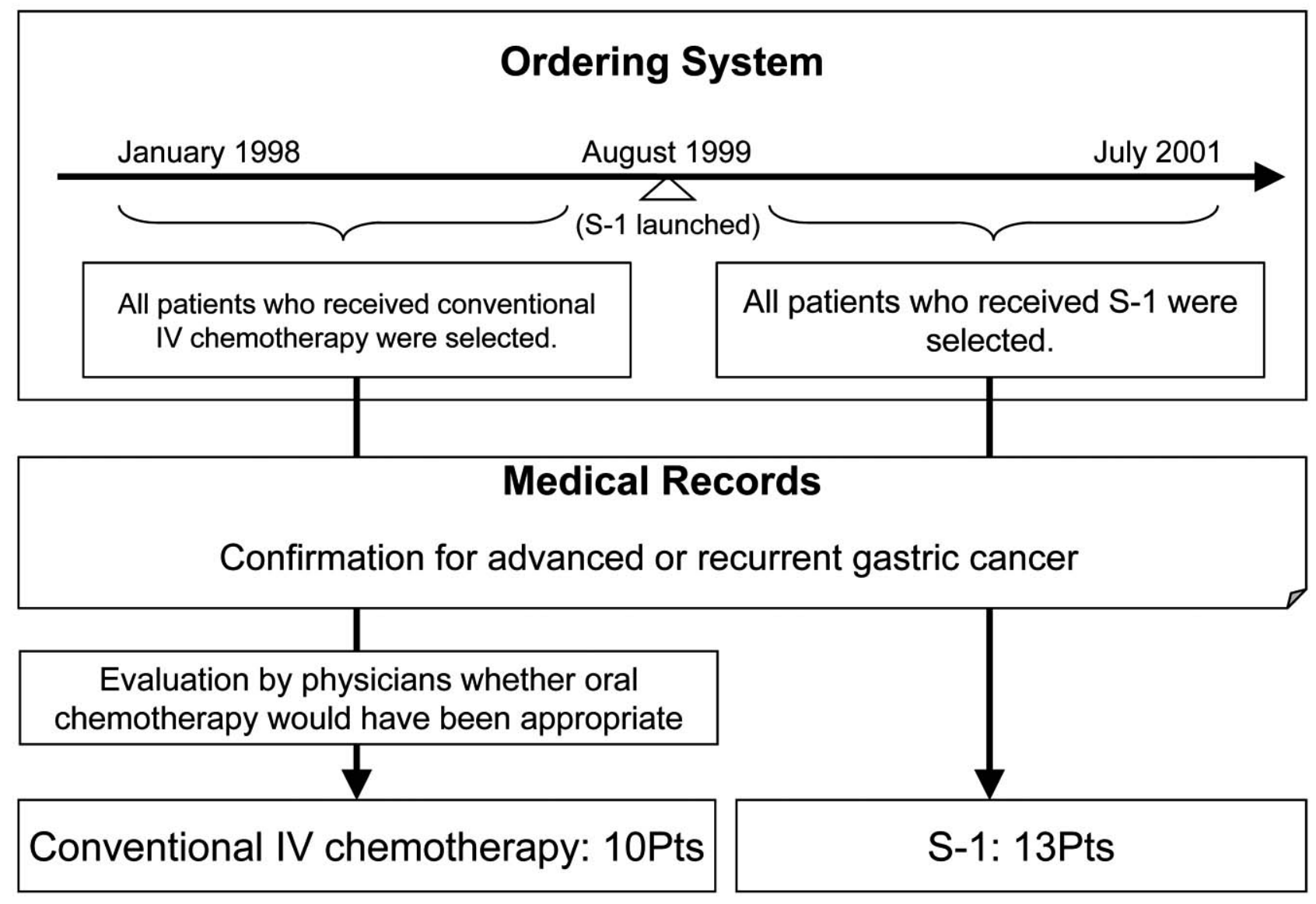

Fig. (1). Patient selection. All patients who received S-1 after August 1999, when S-1 became available at the hospital, were selected. All patients who received injectable preparations of 5-fluorouracil, cisplatin, methotrexate, or irinotecan before August 1999 were selected. 
Drugs were classified into the subcategories of anticancer agents, granulocyte colony-stimulating factor (G-CSF), antiemetic, blood-derived preparations (included in transfusion), and other drugs. Monthly average costs were calculated for the diagnostic period (from 1 month before the start of chemotherapy to the month of starting chemotherapy), the chemotherapy period, and the terminal care period (from 1 month before death to the month of death) and statistically compared between the groups using the Mann-Whitney U-test.

\section{Measuring Utilities (Fig. 2)}

\section{Standard Gamble, Time Trade-Off, and Rating Scale Methods [12]}

We identified possible health states by reviewing the medical records of the subjects, and made 27 scenarios each consisting of three levels of mobility, pain, and digestive symptoms, which seem to be typical items describing the health states of gastric cancer patients who receive chemotherapy treatment. The utilities of the 27 scenarios were assessed by 9 oncology pharmacists and 18 oncology nurses, using the standard gamble (SG), time trade-off (TTO), and rating scale (RS) methods. To minimize deviations of estimations among the health-care professionals, we instructed them on how to evaluate utilities by means of SG, TTO, and RS before the estimation. The difference in utility scores between the nurses and pharmacists in our study was small and not significant [13]. Information derived from the medical records of each patient during chemotherapy, diagnosis, and terminal care in both groups was classified into the appropriate scenarios. The utilities of each patient were calculated on the basis of the utility scores of the scenarios and were statistically compared between the S-1 and IV chemotherapy groups using the Mann-Whitney U-test.

\section{EQ-5D Mapping Procedure [14]}

Information derived from medical records, including mobility, meal ingestion, pain, and other symptoms, was mapped into the EQ-5D health states for each patient in both groups. The utilities of each patient were derived from the Japanese tariff of EQ-5D for every month and statistically (using the Mann-Whitney U-test) compared between the S-1 and IV chemotherapy groups.

\section{Sensitivity Analysis}

When conducting the economic evaluations, we assumed that survival was similar for S-1 treatment and conventional intravenous chemotherapy, which might have biased our results. We therefore carried out one-way sensitivity analysis of QALM (quality-adjusted life months) during chemotherapy by varying the difference in MST between the regimens by $0,1,2,3$, and 4 months to test the robustness of our data.

\section{RESULTS}

\section{Patient Characteristics}

Thirteen patients who received S- 1 and 10 who received conventional intravenous chemotherapy from January 1998 through July 2001 were identified from the patient database of Showa University Hospital.

Patient characteristics in both groups are shown in Table 2. The two groups were well balanced with respect to clinical characteristics except sex. The average age was 62.5 years in S-1 group and 62.1 years in IV chemotherapy group. The cancer type was advanced in 5 patients and recurrent in 8 patients in the S-1 group and advanced in 4 and recurrent in 6 in the IV chemotherapy group. The regimens received by the IV chemotherapy group were low-dose 5-fluourouracil plus cisplatin (FP) in 7 patients, methotrexate plus 5fluorouracil $(\mathrm{MTX} / 5 \mathrm{FU})$ in 1 , irinotecan plus cisplatin

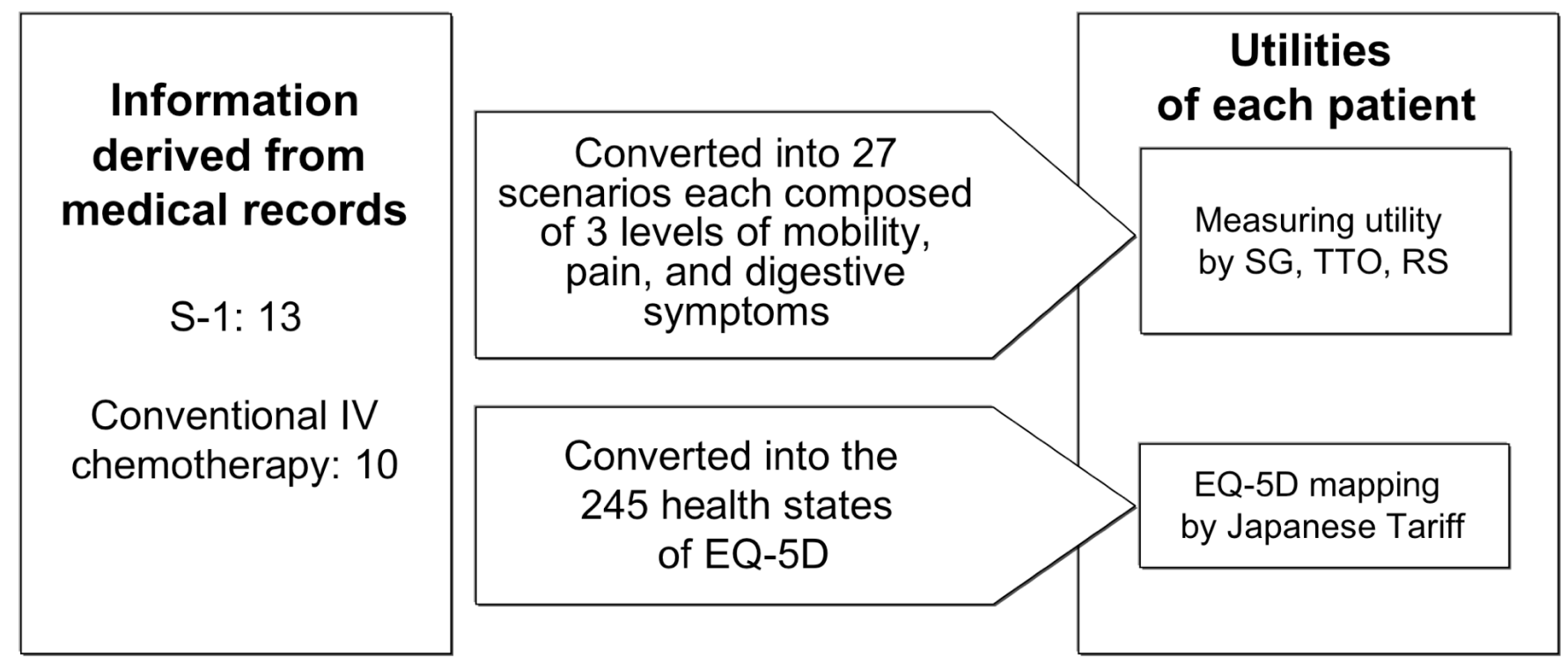

Fig. (2). Measuring utilities. Standard gamble, time trade-off, and rating scale methods: Information derived from medical records was classified into the appropriate scenarios. The utilities of each patient were calculated on the basis of the utility scores of the scenarios. $E Q-5 D$ mapping procedure: Information derived from medical records was mapped into the EQ-5D health states for each patient in both groups. The utilities of each patient were derived from the Japanese tariff of EQ-5D. 
Table 2. Patient Characteristics

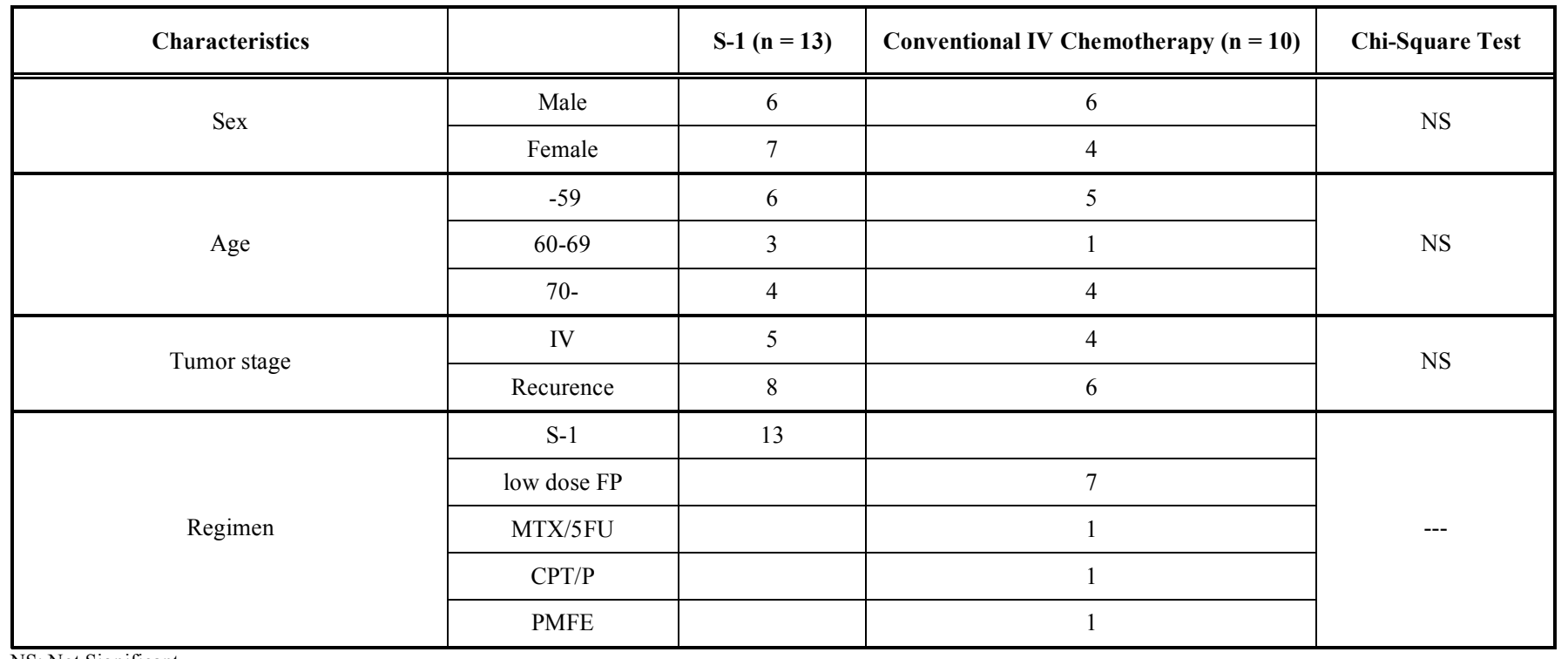

(CPTP) in 1 and a combination of 5-fluorouracil, cisplatin, mitomycin $\mathrm{C}$, and etoposide (PMFE) in 1.

\section{Costs}

The costs during diagnosis, chemotherapy, and terminal care are shown in Table 3 and Fig. (3). The average ( \pm SE) monthly total costs during chemotherapy were significantly lower in the S-1 group $(327,640 \pm 47,647$ yen) than in the conventional chemotherapy group $(852,874 \pm 62,412$ yen) (the Mann-Whitney U-test: $\mathrm{P}<0.05$ ), which resulted in saving the cost of 525,233 yen per patient. During the diagnosis period and terminal care period, the average monthly costs were respectively 507,790 yen and 603,582 yen in S-1 group, and 592,331 yen and 585,542 yen in IV chemotherapy group. These costs were similar in the groups.

\section{Chemotherapy Drug Costs}

The average monthly drug acquisition cost for chemotherapy per patient was 78,296 yen in the S-1 group and 45,135 yen in the conventional chemotherapy group. The tariff price of S-1 is more expensive than those of lowdose FP, typical conventional intravenous chemotherapy, consisted of " 5 -fluorouracil $350 \mathrm{mg} / \mathrm{m}^{2}$ on days $1-28$ plus cisplatin $10 \mathrm{mg} / \mathrm{m}^{2}$ on days $1-5$ of a 28 -day cycle." Because the dosage of S-1 was "tegafur $80-120 \mathrm{mg} /$ patient on days 1 28 of a 42-day cycle," the monthly drug cost in the S-1 group varied according to the timing of treatment.

\section{Hospitalization Costs}

The monthly average cost of hospitalization to receive chemotherapy was $244,277(37,482$ - 394,285) yen during the first 3 months of treatment in the S-1 group. Following their discharge, patients received $\mathrm{S}-1$ on an outpatient basis, and the cost was $7,412(6,500-7,668)$ yen per month. The monthly average cost of hospitalization was 443,691 $(181,856$ - 506,496) yen in the IV chemotherapy group.

\section{Supportive Treatment Costs}

The average monthly costs for supportive treatment with antiemetics, G-CSF, and blood-derived preparations were respectively 382 yen, 598 yen, and 1,568 yen in the S-1 group, as compared with 44,322 yen, 19,564 yen, and 19,832 yen in the IV chemotherapy group. Costs were lower in the S-1 group than in IV chemotherapy group because of the lower incidence of adverse reactions in the former group.

\section{Utilities}

The utilities during diagnosis, chemotherapy, and terminal care are shown in Table 4 and Fig. (4). Mean $( \pm S E)$ utilities during chemotherapy as measured by the standard gamble method, time trade-off method, rating scale method, and EQ-5D mapping procedure were respectively 0.94 ( \pm $0.01), 0.90( \pm 0.02), 0.90( \pm 0.02)$, and $0.84( \pm 0.02)$ in the S1 group, as compared with $0.79( \pm 0.02), 0.68( \pm 0.03), 0.67$ $( \pm 0.03)$, and $0.52( \pm 0.04)$ in the IV chemotherapy group. The mean utilities in the S-1 group were significantly higher than those in the IV chemotherapy group by all four techniques (the Mann-Whitney U-test: $\mathrm{P}<0.05$ ). The utilities of S-1 during outpatient basis ranged from 0.9 to 1.0 , similar to those of healthy individuals. The utility scores estimated by the EQ-5D mapping procedure were lower than those derived by the other direct methods in both groups. During the diagnosis and terminal care periods, the mean utilities were respectively $0.81-0.93$ and $0.24-0.67$ in the S-1 group, and $0.81-0.93$ and $0.34-0.71$ in the IV chemotherapy group. Utilities during diagnosis and terminal care were similar in the groups.

\section{Sensitivity Analysis}

The results of sensitivity analysis indicated that QALM in the S-1 group was higher than that in the IV chemotherapy group for up to 1 month by the standard gamble method and time trade-off method, 2 months by the rating scale method, and 3 months by the EQ-5D mapping procedure (Table 5). 
Table 3. Average Monthly Costs (Yen)

\begin{tabular}{|c|c|c|c|c|c|c|c|c|c|c|c|}
\hline & \multicolumn{9}{|c|}{ During Chemotherapy } & \multirow[b]{2}{*}{ Diagnosis } & \multirow{2}{*}{$\begin{array}{c}\text { Terminal } \\
\text { Care }\end{array}$} \\
\hline & $\begin{array}{c}\text { 1st } \\
\text { Month }\end{array}$ & \begin{tabular}{|c|} 
2nd \\
Month
\end{tabular} & \begin{tabular}{|c|} 
3rd \\
Month
\end{tabular} & \begin{tabular}{|c|} 
4th \\
Month
\end{tabular} & \begin{tabular}{|c|} 
5th \\
Month
\end{tabular} & $\begin{array}{l}\text { 6th } \\
\text { Month }\end{array}$ & $\begin{array}{c}\text { 7th } \\
\text { Month }\end{array}$ & \begin{tabular}{|c} 
8th \\
Month
\end{tabular} & $\begin{array}{l}\text { All of Months } \\
\quad( \pm \text { SE) }\end{array}$ & & \\
\hline \multicolumn{12}{|l|}{ S-1 } \\
\hline Total & 684,860 & 397,803 & 185,970 & 122,547 & 140,163 & 126,989 & 111,604 & 86,100 & $327,640 \pm 47,647$ & 507,790 & 603,582 \\
\hline Hospitalization and physician consultation & 394,285 & 207,310 & 37,482 & 7,668 & 7,597 & 7,400 & 7,106 & 6,500 & $147,598 \pm 33,842$ & 271,308 & 360,024 \\
\hline Drugs and administration & 141,008 & 121,573 & 101,998 & 60,909 & 99,587 & 89,786 & 79,696 & 71,150 & $107,408 \pm 9,784$ & 93,617 & 96,640 \\
\hline Operations and procedures & 918 & 6,474 & 0 & 0 & 0 & 5,220 & 0 & 0 & $1,833 \pm 1,144$ & 9,420 & 84,628 \\
\hline Laboratory and diagnostic tests & 147,257 & 59,598 & 44,616 & 53,582 & 32,979 & 23,763 & 24,802 & 8,450 & $69,537 \pm 10,735$ & 130,260 & 56,241 \\
\hline Others & 1,392 & 2,849 & 1,875 & 388 & 0 & 820 & 0 & 0 & $1,264 \pm 398$ & 3,186 & 6,049 \\
\hline Anti-cancer agent (S-1) & 62,711 & 95,005 & 93,273 & 54,979 & 92,400 & 83,863 & 73,277 & 65,722 & $78,296 \pm 4,605$ & & \\
\hline Anti-emetics & 0 & 2,343 & 0 & 0 & 0 & 0 & 0 & 0 & $382 \pm 378$ & & \\
\hline G-CSF & 2,252 & 0 & 0 & 0 & 0 & 0 & 0 & 0 & $598 \pm 413$ & & \\
\hline Blood transfusion & 0 & 6,348 & 0 & 0 & 0 & 5,208 & 0 & 0 & $1,568 \pm 1,142$ & & \\
\hline \multicolumn{12}{|l|}{ IV chemotherapy } \\
\hline Total & 879,237 & 961,272 & 760,341 & 503,273 & 687,846 & $1,028,908$ & & & $852,874 \pm 62,412$ & 592,331 & 585,542 \\
\hline Hospitalization and physician consultation & 462,609 & 506,496 & 434,920 & 181,856 & 259,845 & 669,748 & & & $443,691 \pm 33,404$ & 335,017 & 326,626 \\
\hline Drugs and administration & 243,668 & 251,597 & 184,245 & 186,870 & 188,639 & 231,831 & & & $230,194 \pm 19,664$ & 85,393 & 137,606 \\
\hline Operations and procedures & 12,587 & 21,119 & 50,619 & 0 & 107,015 & 1,834 & & & $25,482 \pm 9,013$ & 5,964 & 45,276 \\
\hline Laboratory and diagnostic tests & 158,343 & 152,026 & 85,461 & 57,033 & 112,737 & 123,605 & & & $135,353 \pm 14,287$ & 159,739 & 70,208 \\
\hline Others & 2,029 & 30,034 & 5,096 & 77,514 & 19,609 & 1,890 & & & $18,153 \pm 9,442$ & 6,219 & 5,826 \\
\hline Anti-cancer agents & 47,789 & 37,062 & 51,892 & 58,744 & 36,740 & 52,466 & & & $45,135 \pm 4,448$ & & \\
\hline Anti-emetics & 50,131 & 35,424 & 60,936 & 39,648 & 32,391 & 40,770 & & & $44,322 \pm 7,824$ & & \\
\hline G-CSF & 18,008 & 28,492 & 0 & 0 & 50,319 & 0 & & & $19,564 \pm 7,192$ & & \\
\hline Blood transfusion & 6,711 & 18,551 & 28,287 & 0 & 107,620 & 0 & & & $19,832 \pm 8,550$ & & \\
\hline
\end{tabular}

G-CSF: granulocyte-colony stimulating factor.

Anti-cancer agents, anti-emetics and G-CSF were included in "Drugs and administration".

Blood transfusion was included in "Operations and procedures".

Diagnosis: from 1 month before the start of chemotherapy to the month of starting chemotherapy.

Terminal care: from 1 month before death to the month of death.

The exchange rate as of November 11, 2008 is 98 yen per U.S. dollar.

\section{DISCUSSION}

The Japanese National Health Insurance fee-for-service system has been used nationwide since the system was introduced in 1961. In 2003, however, new Diagnosis Procedure Combination (DPC) prospective payment systems, similar to Diagnosis Related Group/Prospective Payment System (DRG/PPS), were introduced at university hospitals. Hospital administrators and physicians thus became more aware and interested in health-care costs, and economic evaluations are assuming greater importance. Costs for cancer treatment are now closely scrutinized because of the frequent use of new expensive drugs, such as molecular-targeting agents.

Furthermore, the rapid growth of the elderly population and the attendant increase in the number of patients with cancer has led to enactment of the new law, "The Basic Act on Anti-Cancer Measures" in Japan. Since the new law emphasizes the QOL of patients receiving cancer treatment, the assessment of QOL has attracted attention.

We conducted this cost-utility analysis against this background of increasing emphasis on cost effectiveness and patients' QOL. Our main objective was to compare the costrelated benefits of oral S-1 with those of conventional intravenous chemotherapy in patients with advanced or recurrent gastric cancer. Our results showed that oral S-1 was associated with higher cost effectiveness and a better QOL than was conventional intravenous chemotherapy.

The use of conventional intravenous chemotherapy was accompanied by higher levels of various other costs, such as expenses for hospitalization and laboratory and diagnostic tests, as compared with oral S-1. Costs for supportive treatment for the prevention and treatment of adverse effects of anticancer drugs, including costs for antiemetics, G-CSF, and blood-derived preparations, were also higher for conventional intravenous chemotherapy. On the other hand, 


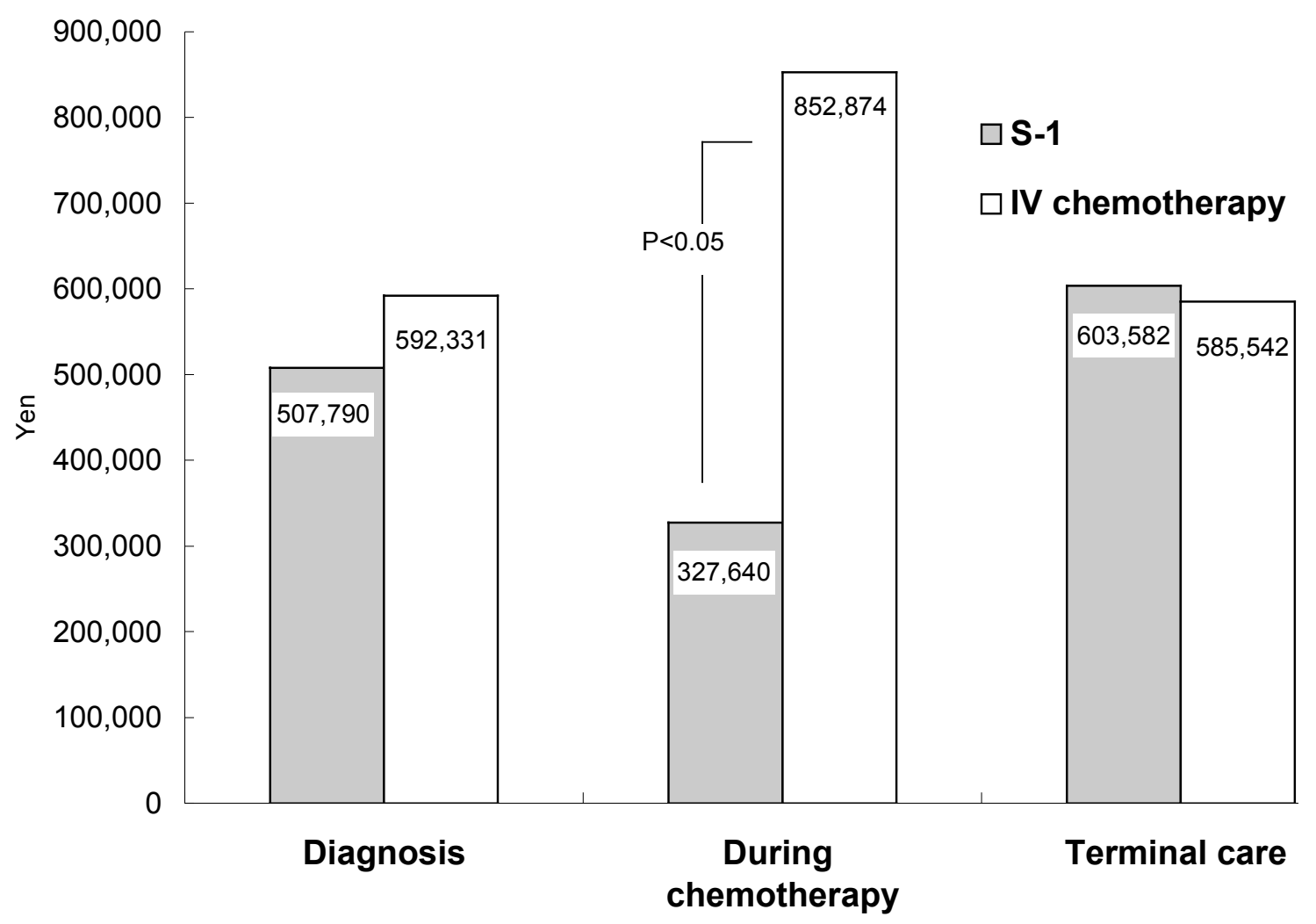

Fig. (3). Total costs (monthly average costs). Diagnosis: from 1 month before the start of chemotherapy to the month of starting chemotherapy. Terminal care: from 1 month before death to the month of death. P value was calculated with the use of the Mann-Whitney U-test.

Table 4. Average Utilities for Each Month During Chemotherapy

\begin{tabular}{|c|c|c|c|c|c|c|c|c|c|c|c|}
\hline & $\begin{array}{c}\text { 1st } \\
\text { Month }\end{array}$ & $\begin{array}{c}\text { 2nd } \\
\text { Month }\end{array}$ & $\begin{array}{c}\text { 3rd } \\
\text { Month }\end{array}$ & $\begin{array}{c}\text { 4th } \\
\text { Month }\end{array}$ & $\begin{array}{c}\text { 5th } \\
\text { Month }\end{array}$ & $\begin{array}{l}\text { 6th } \\
\text { Month }\end{array}$ & $\begin{array}{c}\text { 7th } \\
\text { Month }\end{array}$ & $\begin{array}{c}\text { 8th } \\
\text { Month }\end{array}$ & $\begin{array}{l}\text { All of Months } \\
\quad( \pm \text { SE })\end{array}$ & Diagnosis & $\begin{array}{c}\text { Terminal } \\
\text { Care }\end{array}$ \\
\hline \multicolumn{12}{|l|}{ S-1 } \\
\hline SG & 0.88 & 0.96 & 0.98 & 0.96 & 0.99 & 0.96 & 0.94 & 1.00 & $0.94 \pm 0.01$ & 0.93 & 0.67 \\
\hline TTO & 0.80 & 0.93 & 0.95 & 0.92 & 0.97 & 0.93 & 0.89 & 0.99 & $0.90 \pm 0.02$ & 0.88 & 0.50 \\
\hline RS & 0.79 & 0.93 & 0.95 & 0.92 & 0.97 & 0.93 & 0.88 & 0.99 & $0.90 \pm 0.02$ & 0.88 & 0.49 \\
\hline EQ-5D & 0.66 & 0.88 & 0.93 & 0.90 & 0.96 & 0.92 & 0.87 & 0.99 & $0.84 \pm 0.02$ & 0.81 & 0.24 \\
\hline \multicolumn{12}{|c|}{ IV Chemotherapy } \\
\hline SG & 0.79 & 0.73 & 0.83 & 0.83 & 0.87 & 0.93 & & & $0.79 \pm 0.02$ & 0.93 & 0.71 \\
\hline TTO & 0.68 & 0.59 & 0.72 & 0.76 & 0.81 & 0.83 & & & $0.68 \pm 0.03$ & 0.87 & 0.50 \\
\hline RS & 0.67 & 0.57 & 0.70 & 0.74 & 0.79 & 0.82 & & & $0.67 \pm 0.03$ & 0.88 & 0.51 \\
\hline EQ-5D & 0.50 & 0.41 & 0.56 & 0.61 & 0.69 & 0.77 & & & $0.52 \pm 0.04$ & 0.81 & 0.34 \\
\hline
\end{tabular}

SG: Standard gamble, TTO: Time trade-off, RS: Rating scale, EQ-5D: EQ-5D mapping procedure.

although patients initially received $\mathrm{S}-1$ in the hospital to ensure safety, S-1 was given on an outpatient basis after the second course of treatment because of the higher safety and convenience of oral treatment.

Economic analyses for gastric cancer are few in the world. One of such study was performed by Norum et al. [15]. The regimens they compared in the study were injectable treatments, FAM (5FU, adriamycin, mitomycin C) and ELF (etoposide, leucovorin, 5FU). The monthly costs of those regimens were from 82,000 to 440,000 yen.

The QOL of patients in our study was evaluated by nurses and pharmacists who were involved in cancer treatment because it is difficult to directly ask patients with cancer about their QOL. Although oncology professionals may not appropriately evaluate potential toxicities and emotional difficulties that patients might be experiencing, health-care professionals seems to have the best 


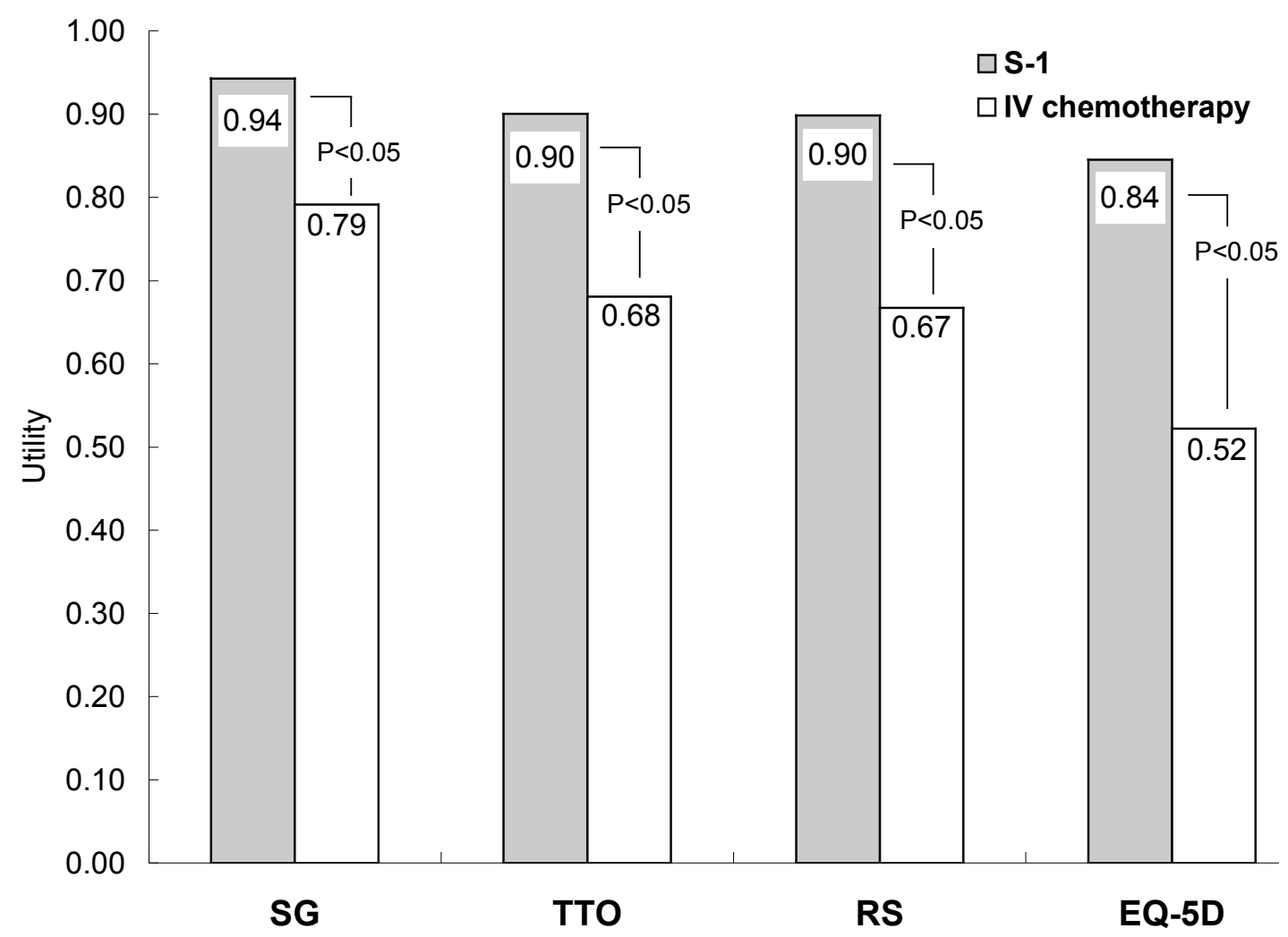

Fig. (4). Utilities for each method. SG: Standard gamble, TTO: Time trade-off, RS: Rating scale, EQ-5D: EQ-5D mapping procedure. P values were calculated with the use of the Mann-Whitney U-test.

understanding of the disease and the described health state. A systematic review of cost-utility assessments in oncology by Earl et al. showed that health-care professionals were the most common sources for utility estimates [16].

Table 5. Sensitivity Analysis

\begin{tabular}{|c|c|c|c|c|}
\hline \multirow{2}{*}{ MST Difference (Months) } & \multicolumn{4}{|c|}{ QALM Difference } \\
\cline { 2 - 5 } & SG & TTO & RS & EQ-5D \\
\hline \hline 0 & 1.20 & 1.76 & 1.84 & 2.56 \\
\hline-1 & 0.26 & 0.86 & 0.94 & 1.72 \\
\hline-2 & -0.68 & -0.04 & 0.04 & 0.88 \\
\hline-3 & -1.62 & -0.94 & -0.86 & 0.04 \\
\hline-4 & -2.56 & -1.84 & -1.76 & -0.8 \\
\hline
\end{tabular}

In this study the utility scores were low for conventional intravenous chemotherapy, most likely because the patients were hospitalized for treatment and confined to bed during continuous intravenous infusions. Side effects such as nausea, vomiting, and general fatigue probably also decreased the utility scores in IV chemotherapy group. In contrast, S-1 was given gradually on an outpatient basis after the first course of treatment, did not require confinement to bed, and had milder side effects. These factors most likely resulted in a better QOL in the patients given S-1, comparable to that of healthy individuals.

According to the league table of the utility scores [17], the utility of oral anticancer agents has not been evaluated previously in gastric cancer. Our results will hopefully provide useful information to physicians who treat patients with advanced or recurrent gastric cancer.

Our study had several limitations. First, this was a retrospective study of a small number of patients. Second, the patients in the S-1 group and conventional chemotherapy group were treated during different time periods. To minimize bias, however, our data was based on the records of patients with advanced or recurrent gastric cancer who actually received the study treatments, and the clinical characteristics of the groups were well balanced. Despite the small numbers of patients, our results showed that oral S-1 was associated with lower treatment-related costs and higher utility scores, reflecting a better QOL as compared with the IV chemotherapy group. The results of sensitivity analysis indicated the robustness of our findings.

To address the societal implications of treatment with S-1 versus conventional intravenous chemotherapy, we estimated all assessable health-care costs, including costs based on the Japanese fee-for-service system and costs paid directly by patients, such as extra-charge bed and meal charges. The inclusion of transportation expenses and costs related to lost productivity were beyond the scope of our study. These factors could be addressed in future cost analyses.

Because our data were derived from the medical records of actually treated patients, we did not perform sensitivity analysis of cost parameters. However, the recent introduction of cost-containing measures in Japan will most likely lead to increasing numbers of patients who receive injectable preparations on an outpatient basis. When we compared the 
groups after subtracting hospitalization costs, however, S-1 was still more cost effective than conventional intravenous chemotherapy (about 500,000 yen), because supportive costs for the prevention and treatment of adverse events remained high for the latter. QOL was considered to be also still better in the patients who received S-1 than in those who received conventional intravenous chemotherapy which might require confinement to bed even on an outpatient basis.

Since gastric cancer is the most frequent type of cancer in Japan, by extrapolating our findings to the approximately 50,000 patients who die of gastric cancer per year, we estimate that switching from conventional intravenous chemotherapy to oral S-1 would results in health-care cost savings of 25 billion yen annually.

\section{CONCLUSION}

Our results show that oral S-1 is a dominant strategy with a lower cost, and better health outcomes than conventional intravenous chemotherapy in patients with advanced or recurrent gastric cancer.

\section{ACKNOWLEDGEMENTS}

The authors would like to acknowledge Dr Tsutomu Kaetsu of the Showa University hospital for advice with this study.

\section{REFERENCES}

[1] GLOBOCAN 2002 database. International Agency for Research on Cancer 2002. [Retrieved 2007 Dec 24]. Available from http://www-dep.iarc.fr/

[2] Kato H, Ed. Cancer Statistics in Japan. Tokyo: Foundation for Promotion of Cancer Research 2008; pp. 70-3

[3] National Comprehensive Cancer Network. Gastric cancer (V.2.2007). [Accessed 2007 Dec 24]. Available at http://www. ncen.org

[4] Sasaki T, Ed. Gastric Cancer Treatment Guideline in Japan, 2nd ed. Tokyo: Kanehara 2004; pp. 34-5

[5] Sakata Y, Ohtsu A, Horikoshi N, et al. Late phase II study of novel oral fluoropyrimidine anticancer drug S-1 in advanced gastric cancer patients. Eur J Cancer 1998; 34: 1715-20.

[6] Koizumi W, Kurihara M, Nakano S, et al. Phase II study of S-1, novel oral derivative of 5-fluorouracil, in advanced gastric cancer. Oncology 2000; 58: 191-7.
[7] Boku N, Yamamoto S, Shirao K, et al. Randomized phase III study of 5-fluorouracil (5-FU) alone versus combination of irinotecan and cisplatin (CP) versus S-1 alone in advanced gastric cancer (JCOG9912). Proceedings of American Society of Clinical Oncology. Chicago, Illinois 2007; Abstract LBA4513.

[8] Saif MW, Syrigos KN, Katirtzoglou NA. S-1: A promising new oral fluoropyrimidine derivative. Expert Opin Investig Drugs 2009; 18: $335-48$.

[9] Tanaka K, Kaetsu T, Suzuki S, et al. Pharmacoeconomic study of chemotherapy for gastric cancer: analysis of medical costs for oral fluoropyrimidine TS-1 and conventional i.v therapy. Gan To Kagaku Ryoho 2003; 30: 73-80.

[10] Sakamaki H, Ikeda S, Yajima S, et al. Cost analysis of treatment for advanced or recurrent gastric cancer in japan: economic comparison between the oral fluoropyrimidine TS-1 and conventional intravenous chemotherapy. Value Health 2003; 6: 736: PCN15.

[11] Sakamaki H, Ikeda S, Yajima S, et al. Cost-utility analysis of chemotherapy in advanced or recurrent gastric cancer: oral fluoropyrimidine TS-1 versus conventional intravenous chemotherapy. Value Health 2005; 8: A43: PCN33.

[12] Drumond MF, O'Brien BJ, Stoddart GL, Torrane GW. Methods for the economic evaluation of health care programmes. New York: Oxford University Press 1997.

[13] Shimizu H, Tanaka K, Ikeda S, et al. Utility-based evaluation of the quality of life of patients with gastric cancer who receive chemotherapy. Yakugaku Zasshi 2008; 128: 783-93.

[14] Gold MR, Siegel JE, Russell LB, et al. Cost-effectiveness in health and medicine. New York: Oxford University Press 1996.

[15] Norum J, Angelsen V. Chemotherapy in gastric cancer: an economic evaluation of the FAM versus ELF regimens. J Chemother 1995; 7: 455-9.

[16] Earl CC, Chapman RH, Bakere CS, et al. Systematic overview of cost-utility assessments in oncology. J Clin Oncol 2000; 18: 330217.

[17] Catalog of Preference Scores. New England Medical Center. [Retrieved 2007 Dec 24] Available from: http://research.tuftsnemc.org/cear/

[18] Ohtsu A, Shimada Y, Yoshida S, et al. Phase II study of protracted infusional 5-fluorouracil combined with cisplatinum for advanced gastric cancer: report from the Japan Clinical Oncology Group (JCOG). Eur J Cancer 1994; 30A: 2091-3.

[19] Murakami M, Ota K, Miyazaki T, et al. Sequential methotrexate-5fluorouracil (MTX-5-FU) treatment of patients with advanced gastric and colorectal cancer. Sequential Methotrexate-5-FU Study Group. Gan To Kagaku Ryoho 1987; 14: 2482-90.

[20] Boku N, Ohtsu A, Shimada Y, et al. Phase II study of a combination of irinotecan and cisplatin against metastatic gastric cancer. J Clin Oncol 1999; 17: 319-23. 